\title{
TBM
}

\section{Implementing an evidence-based breast cancer support and communication tool to newly diagnosed patients as standard care in two institutions}

\author{
Alanna Kulchak Rahm, PhD, MS, CGC, ${ }^{1,2}$ Robert P. Hawkins, PhD, ${ }^{3}$ James W. Dearing, PhD, ${ }^{4}$ \\ Suzanne Pingree, PhD, Jana Bolduan Lomax, PsyD, ${ }^{6}$ Helen McDowell, MS, ${ }^{5}$ Erica Ferro Morse, MA, ${ }^{2}$ \\ BreAnne Barela, BA $^{2}$
}

Geisinger Health System,

Genomic Medicine Institute, $100 \mathrm{~N}$.

Academy Blvd, Danville, PA 17822,

USA

${ }^{2}$ Institute for Health Research,

Kaiser Permanente Colorado,

Denver, CO, USA

${ }^{3}$ School of Journalism and Mass Communication,

University of Wisconsin, Madison, WI, USA

${ }^{4}$ Department of Communications, Michigan State University, East

Lansing, MI, USA

${ }^{5}$ Center for Health Enhancement

Systems Studies,

University of Wisconsin, Madison, WI, USA

${ }^{6}$ Comprehensive Cancer Center, Exempla Saint Joseph Hospital and

Exempla Good Samaritan Medical

Center, Denver, CO, USA

Correspondence to: A Rahm

akrahm@geisinger.edu

Cite this as: TBM 2015;5:198-206 doi: 10.1007/s13142-015-0305-4

\section{Abstract}

While many women turn to the Internet to obtain health information, it is unlikely that unstructured Internet use provides optimal benefit to women newly diagnosed with breast cancer, due to uneven quality, conflicting claims, redundancy, and search engine idiosyncrasies, which may make finding information and assessing its accuracy and applicability difficult. To answer the need for information and support, the Comprehensive Health Enhancement Support System (CHESS) was developed to provide access to integrated information for decisionmaking, behavior change, and emotional support, and has been validated in randomized trials. This observational study of real-world implementation focuses on the process of integrating CHESS into standard care in two Denver healthcare systems. Results from this study provide guidance for implementation of other web-based patient information and support programs in large healthcare organizations.

\section{Keywords}

eHealth, Implementation, Dissemination, Communication, Breast cancer support

\section{INTRODUCTION}

A woman newly diagnosed with breast cancer experiences skyrocketing uncertainty. She is confronted with a daunting technical language, fear and concern both from herself and from loved ones, and thousands of websites, articles, advertisements, and hearsay including the advice and experiences of friends and new acquaintances who have had or currently do have breast cancer. Much of the information and stories to which she is exposed is comforting but also raises new questions, most of them couched in terms of risk and probability. She must learn about her disease and its treatment, cope with emotional distress and interpersonal issues, make decisions about her treatment, and negotiate work and family obligations. While many women turn to the Internet to obtain information [1], it is unlikely that unstructured Internet use provides optimal benefit, due to uneven quality, conflicting

\section{Implications}

Policy: Organizational priorities and changes affect implementation, and the innovation will not be considered a factor in any changes being made if constant communication is not maintained with leadership as well as end-users.

Research: Research on implementation of programs within health systems provides much needed insights into the reasons why certain implementation strategies may or may not work in different settings.

Practice: Creating processes for constant formal and informal communication and objective tracking of events affecting implementation can help with the process of implementation and dissemination of interventions throughout any organization.

claims, and redundancy, among other things [2]. To answer the need for information and support, various Interactive Cancer Communication Systems (ICCS) have been developed over the last 20 years to provide individuals access to integrated information and decision-making, behavior change, and emotional support for health issues.

One such ICCS is the focus of this paper. The Comprehensive Health Enhancement Support System (CHESS) is a web-based resource of integrated services that have been developed by the University of Wisconsin (UW) for a number of conditions, including breast cancer [3]. CHESS was initially created by teams of researchers and clinicians, with input from patients and family members on what resources patients would likely need. CHESS for breast cancer patients was developed by the UW Center of Excellence in Cancer Communication Research (CECCR) and has been validated in a number of randomized controlled trials funded by the National Cancer Institute (NCI). In these prior studies, CHESS has been shown to improve cancer knowledge, social 
support, quality of life, and eHealth-specific use of the Internet $[2,4]$. CHESS use has also been shown to reduce demands on physician time and cost of care [5]. Additionally, some studies have shown that underserved and elderly populations use and benefit equally or more from CHESS use [6, 7]. Furthermore, a substantial body of research evidence indicates that CHESS, and other systems like CHESS, can lead to patients and families who are better informed, more comfortable seeking and using information, less anxious, experience greater social support, and communicate more effectively with health care professionals [8-11].

CHESS is integrated in that all functions reference each other so that the use of one resource leads to and reinforces other resources on the same website. The CHESS breast cancer website includes multiple menudriven and searchable components for easy use regardless of whether a woman is looking for specific information, looking for "what's next", wanting to hear stories from others to know she's not alone, or is looking for local resources. To reduce information load, CHESS includes a step-by-step guide that organizes resources specific to steps in diagnosis and treatment. It also includes an About Breast Cancer menu organized by categories and linking to vetted, updated, comprehensive cancer information on the Internet. Also included is a link to a reliable online medical dictionary, searchable survivor stories in audio only or audio-visual format, a blog of timely posts by CHESS staff pertinent to breast cancer care to which CHESS users can post comments, and links to local resources and an event calendar. CHESS also includes the ability to "Ask an Expert" which sends a secure message directly to a cancer support specialist who responds within $24 \mathrm{~h}$, and a "just chatting" function that allows users to send secure email messages to other CHESS users.

There are important reasons to integrate CHESS and similar systems into healthcare organizations. Rather than relying on patients to find high-quality online support systems amid the cacophony of uneven or profit-motivated and untested resources, healthcare systems are ideally positioned to identify and communicate with patients at key moments of need, such as at initial diagnosis. Encouragement and direction from clinical staff could also help focus and direct use, making CHESS more effective [12].

However, disseminating evidence-based innovations into healthcare organizations and supporting high-quality implementation are major steps that present significant challenges. Therefore, adoption of the breast cancer CHESS ICCS by a healthcare organization only begins [13] a process that relies heavily on insights from theory and research on implementing change in organizations $[14,15]$. After an organization decides to try an innovation like CHESS, a decision often made by superiors for implementation by subordinates, successful implementation is influenced by a set of individual characteristics (presence, prestige and energy of champions, and opinion leaders) and organizational characteristics (openness to creativity, shared decision-making, and structural and resource support for the intervention) [15]. Likewise, organizational implementation of CHESS or any eHealth innovation is itself only part of achieving use. Provider and patient knowledge and perceptions both of their disease and of the innovation (which is dependent upon communication efforts) will likely help determine the proportion of patients who try, adopt, and sustain their use of the resource. So an innovation like breast cancer CHESS has to support the organization's mission and help to achieve its priorities, fit with the skills and needs of care providers, and be understood by patients as providing real value in order to achieve sustained use and benefit.

With support from the NCI, researchers from two of its Centers of Excellence in Cancer Communication Research (CECCRs) combined to offer CHESS to all newly diagnosed breast cancer patients at two Denver healthcare organizations: Kaiser Permanente Colorado (KPCO), a large $\mathrm{HMOl}$; and Exempla Healthcare (EHC), a 140-year-old community and teaching hospital that serves a larger proportion of minorities. Leaders of each organization agreed to make CHESS part of standard care for breast cancer patients and also agreed to maintain CHESS access for future patients after the grant period ended if organizational experience with CHESS was positive.

The overall goals for the CHESS implementation project were to determine the following:

1. How effectively is CHESS offered to patients as part of standard care at each institution?

2. How many women learn about CHESS availability, and do differences in either patient demographics or organizational presentation methods affect awareness of or perceptions of CHESS?

3. What proportion of patients will obtain an access password, use CHESS, and how often?

4. Why do some women learn/register/use and others do not? What are the effects of patient perceptions of needs vs. patient perceptions of resources vs. computer/Internet access vs. clinician perceptions and advocacy? Do disadvantaged women adopt and use CHESS as much as advantaged women?

5. To what extent and for which women will use of CHESS here correspond to "effective use" as previously determined in our efficacy studies?

6. How do staff perceptions and actions affect patient perceptions and adoption of CHESS?

7. What proportion of different staff groups (e.g., surgeons, oncologists, radiologists, care coordinators, patient navigators) advise, recommend, and remind patients about CHESS?

8. Is CHESS use related to patient satisfaction and quality of life, and to clinician satisfaction with patients?

9. How does staff and patient use of CHESS compare to staff and patient use of other resources for 
breast cancer patients? Where does CHESS fit among those resources?

10. Do organizational and unit strategic priorities affect CHESS adoption and use? Do the different practice models at these two institutions (HMO vs. not-for-profit hospital) affect implementation?

The present report focuses solely on the process of actual implementation of CHESS in these two organizations, observation of the barriers and facilitators of integrating the CHESS resource into standard care, and the expected and final process of how CHESS was offered in each institution. We will also report how these experiences inform further dissemination of CHESS to other KP regions and other organizations, and how these observations can guide implementation efforts in healthcare settings.

\section{METHODS}

\section{Implementation tracking}

This is a longitudinal, observational study of implementing a well-tested, evidence-based eHealth resource for breast cancer patients. Data are derived from meeting agendas, meeting notes, and a tracking system developed specifically for understanding CHESS implementation. This implementation tracking system captures meetings, conversations, events, dates, persons involved, the "what happened" (without interpretation) of the event, and the interpretation of the event including the solution/action taken by the research team based on the interpretation.

\section{RESULTS}

This undertaking to implement CHESS began in March 2009, when conversations between several of the authors determined that KPCO could be the site for a real-world implementation of CHESS. Working quickly and with little input from KPCO clinicians, the team submitted a research grant proposal to NCI in June to study the implementation of CHESS at KPCO. Table 1 summarizes the series of experiences encountered, solutions created, and lessons learned during the CHESS implementation process. The following results describe these experiences and solutions in detail.

First, despite organizational preference for a personalized, site-specific implementation study, the initial critique from NCI's study section led us to sharpen the research questions to those outlined above, and to augment the program with a second Denver healthcare organization $(\mathrm{EHC})$ to reach a more diverse patient population to meet the needs of both the institution and the funders. Early spring 2010 saw a series of meetings with $\mathrm{KPCO}$ and $\mathrm{EHC}$ clinical stakeholders (surgeons, oncologists, radiologists, nurses, and psychosocial services) to improve stakeholder input into the project design. Initial presentations to small groups were followed about a month later by more formal presentations to the breast care steering committees at each institution. These meetings demonstrated
CHESS and summarized clinical trial evidence, noting how CHESS met the expressed needs at the institutions, and then moved to discussions of implementation desirability and options. Clinical stakeholders were given a URL and guest password to examine CHESS in as much depth as they desired. Stakeholders determined that CHESS offered certain advantages compared to their standard practice of care. Therefore, CHESS fit with both organizations' values to provide evidence-based care, and filled a need to provide vetted, timely, and medically accurate information to patients without requiring additional staff time.

Additionally, the KPCO clinician stakeholders from a planned breast care center recommended that adoption of CHESS be considered for immediate implementation (i.e., independent of research funding) by higher level administrators within KPCO. To that end, the UW team created several alternative scenarios for implementing CHESS in Denver, including budget figures for updating and localizing CHESS, managing content updates, staffing the "Ask an Expert" function, and updating the Spanish-language CHESS prototype. These presentations also included a brief discussion of pros and cons of each alternative. Presentations to two levels of organizational leaders occurred during summer 2010, and led to the decision to apply to fund CHESS implementation and evaluation at KPCO and EHC through an NCI grant mechanism in lieu of immediate implementation.

In the time between the proposal and the receipt of grant funding in August, 2011 (more than one year), minimal communication was maintained between researchers and the clinics at the two institutions. As the two CECCR teams began to plan for the actual CHESS implementation, we noted several barriers to implementation despite our efforts to conform to principles of the diffusion of innovations model thus far. First, it was clear that the minimal communication efforts made during the period while waiting for funding were not sufficient for maintaining individual and institutional memory of CHESS (Table 1). Meetings with stakeholders who had participated in previous meetings resulted in fresh questioning of CHESS advantages, whether CHESS was still compatible with organizational values, needs, and current practice, and concerns that it would be too disruptive of current operations. In most cases, it was as if we were starting again in presenting CHESS to the organizations and stakeholders to gain support instead of building on already-established relationship.

Other organizational changes we were not aware of at both institutions also occurred during this time. At EHC, study leadership changed and a new site PI not previously involved was added to lead the project. At $\mathrm{KPCO}$, priorities in oncology had changed; new stressors had surfaced including increased caseloads for oncologists; and planning for the opening of new facilities, leading physicians to raise new doubts about CHESS implementation. Therefore, we began marketing CHESS implementation in terms of a "try before 
Table 1 | Implementation experiences, solutions, and lessons learned for future implementation projects

Project experiences and solutions

Organizational issues priorities differ.
Experience: Funder priorities and organizational

- Funders required multiple implementation sites and multiple research questions to increase generalizability.

- Implementing organizations are concerned with implementation specific to their unique environment.

Solutions: Accommodate both funder and organizations.

- Selected second organization willing to implement as part of the same project.

- Identified and engaged clinical and organizational stakeholders at both institutions via multiple presentations and discussions during proposal preparation.

Experience: Cost considerations may trump potential organization implementation issues.

- Utilization of research funding for resource implementation is appealing to organizations by relieving some implementation cost and commitment concerns, increasing organizational interest in implementation.

- Cost of startup defrayed by grant funding was more appealing than potential timeline misalignment issues for organization.

Solutions: Presented options varying in cost of implementing CHESS, including barriers and benefits of grant-supported implementation vs. organizational-funded implementation.

\section{Clinical and staff Experience: Research-funded implementation} issues offers clinical personnel the chance to "try be fore you buy," with little to no personal or organizational risk.

Solutions: Utilizing this marketing approach in multiple key stakeholder conversations improved clinical stakeholder buy-in and willingness to implement resource.

\section{Experience: Time lag between proposal}

submission and funding award may be too long to maintain engagement.

- Organizational decision makers and clinical staff are too busy to maintain interest while awaiting proposal review and funding award.

Solutions: Intermittent communication was maintained with a single key stakeholder prior to funding, however, organizational priorities in breast cancer and the key stakeholder's role changed during this time period.

Experience: Physician and other clinical staff time is a barrier to resource implementation due to competing priorities and organizational initiatives that are unconcerned with project/ funding timelines.

Solutions: Share the workload.

- Implementation management duties were sustained by the research team.

- Staff champions utilized for implementation instead of MDs whenever possible.

Implementation Experience: Implementation initiative must align issues with organizational standard operating
Lessons learned

$\checkmark$ Identify and involve multiple key organizational stakeholders and champions as part of the team to be involved throughout project development and beyond.

$\checkmark$ Limit research-directed conversations.

$\checkmark$ Have numerous discussions focused on how organizational priorities and research/funding priorities may differ and align.

$\checkmark$ Utilizing grant funding may limit organizational stakeholder commitment to project and resource.

$\checkmark$ Include potential organizational change scenarios and effect on intervention in stakeholder conversations. Guide strategic thinking about the impact of future organizational change on implementation by thinking through the waiting time and the effect of organizational change on implementation.

$\checkmark$ Define future expectations and long-term resource preservation strategies.

$\checkmark$ Organizational commitment to maintain resource after implementation funding ends is required for clinical buy-in.

\section{$\checkmark$ Maintain consistent communication with multiple stakeholders across organizations and departments, and timelines.}

$\checkmark$ Key personnel change over time as organizational priorities change.

$\checkmark$ Staffing shortages limit attention to the intervention; staff focuses on essential responsibilities.

$\checkmark$ Utilize multiple communication strategies such as email, texting, web, telephone and informal 1:1.

$\checkmark$ MDs limited by time will not participate if given choice.

$\checkmark$ Limiting involvement of or dependence on MD and other stakeholders "too busy" may increase organizational participation and implementation success.

$\checkmark$ Utilize staff champions as much as possible.

$\checkmark$ Align resource implementation with existing
organizational standard operating procedures.
$\checkmark$ Solicit and respond to stakeholder feedback.


procedures to minimize work flow disruption for clinical participation.

- Clearly define stakeholder roles and responsibilities.

- Create simple tools to facilitate resource integration and use.

Solutions: Used existing processes to implement resource.

- Defined staff champions and processes to minimize reliance on MDs or new workflows.

- Solicited feedback and developed tools requested by each site to facilitate resource integration and use by all clinical stakeholders.

Experience: Formal communication processes for project evaluation are not sufficient to maintain clinical participation in implementation or provide continual feedback necessary for response to implementation issues.

Solutions: Set communication expectations, including continual informal communication with multiple organizational stakeholders and champions.

- Define formal and informal communication systems (who, when, how) at the start of the project.

- Research staff member kept in constant communication with implementation champions.

- Formal interviews also led to changes in resource implementation procedures. $\checkmark$ Focus on working with staff champions and their workflows.

$\checkmark$ Create and provide tools as requested by all stakeholders.

\begin{abstract}
$\checkmark$ Stakeholder feedback is critical to successful implementation.

$\checkmark$ Define formal and informal communication processes to encourage communication between organizational staff and implementation research team.

$\checkmark$ Utilize all communication processes to identify opportunities for implementation improvement. $\checkmark$ Define response procedures to problems that arise during implementation.

$\checkmark$ Understand that a federally funded intervention may be considerably more important to researchers than it is to practitioners.
\end{abstract}

you buy" to allow for physicians and staff to experience CHESS without commitment. We also identified staff champions among the nurse navigators and care coordinators for whom CHESS fulfilled an unmet need, fit with values, and provided an advantage over services these staff members were currently providing. These individuals also championed CHESS to other departments involved in breast cancer care and showing how CHESS still fit despite the organizational changes.

Additional issues surfacing when CHESS was reintroduced to physicians at KPCO and EHC included the degree to which it was up-to-date and the process of review became an issue. With breast cancer care at one of the participating institutions in turmoil due to staff departures and uncertainty over the future structure of breast care at the institution, a number of doctors were quite hesitant about a long-term commitment to participating in initial and future content reviews. This stimulated us to undertake a major and overdue redesign of breast cancer CHESS. Because other online resources had improved so much since CHESS was originally created, we turned the existing, needs-driven and well-tested structure of the system into a portal. Instead of menu choices leading to content created and maintained at CHESS, those menu choices instead linked to what our staff and clinician advisers judged to be the best resources in each case (e.g., Cancer.gov, ACS, Mayo, etc.). With this change, the information in CHESS still provided the same coverage as before, and was still at the same top level of quality, but is updated more frequently by others instead of CHESS staff and physician volunteers.
Furthermore, we made another structural change that we hoped would be more obvious and more beneficial to breast cancer patients. In the early years of CHESS, the Discussion Group (an asynchronous bulletin board) was very heavily used, sometimes accounting for over half of all time on CHESS. Use of the Discussion Group also stimulated use of other portions of CHESS such as seeking other resources. However, in recent years, we noted decreased use of the Discussion Group, perhaps because of the support alternatives provided by social media and breast cancer-specific online communication resources. We therefore replaced Discussion Group with a blog of commentary on breast cancer issues and news and placed it in a central position on the home screen. Doctors at the two institutions were reluctant to allow patients to initiate blogs because of accuracy concerns, so all blog posts (at least several each week) were created by project staff, with clinicians and patients welcome to comment.

\section{CHESS trial ability and observation of results}

The ability to try out CHESS was important to stakeholders at both institutions. During the discussions of CHESS implementation options, the ability to evaluate the fit of CHESS in the real-world clinical setting was as important as budget considerations to the decision to seek funding as an implementation study. During the reengagement process after funding was received, stakeholders at both institutions expressed concerns about needing organizational support to 
maintain CHESS after the study period. Reassurance was provided that the evaluation process would provide concrete data for the organization to promote sustainability. We reframed the implementation of CHESS as a study without commitment on the clinicians' side but with resources and commitment from the organizations should they wish to maintain CHESS in standard care.

Because of the issues faced from lack of communication with organizational stakeholders between application and funding, the expectation of regular communication through formal and informal processes was established. The purpose of these communication processes was to provide continual feedback about CHESS implementation to stakeholders, as well as for stakeholders to provide input about CHESS to the evaluation team as part of the observational data on implementation. At KPCO, informal check-ins were established as weekly to monthly telephone contacts with care coordinators, and monthly to quarterly or as-needed phone conversations with managers. At $\mathrm{EHC}$, the site implementation lead is a clinician embedded within the care delivery system; therefore, frequent informal communication about CHESS was maintained through department meetings and regular clinical interactions. Formal communication in the form of CHESS quarterly newsletters were also provided to all stakeholders. Newsletters included contact information for study staff, updates on patient utilization of CHESS, updates on evaluation of CHESS through patient interviews and physician surveys, as well as quotations from patients about the usefulness of CHESS and quotes from patients expressing thanks or appreciation for the care they received from specific physicians and staff.

Implementation and sustainability of CHESS as standard care The reengagement of stakeholders, identification of new champions, and redesign of the CHESS website lasted from September 2011 through June 2012. In June 2011, meetings with all stakeholders were conducted to present the redesigned website, to finalize processes for implementing CHESS as standard care for breast cancer patients (with clinicians and staff importantly contributing to identify patient communication opportunities in normal clinical workflow), and to establish ongoing formal and informal communications between the clinic staff and the evaluation team. At these meetings, we framed the implementation of CHESS as a real-world clinical introduction of a product, not a research study, and encouraged use of the informal and formal communication processes to facilitate problem solving and ongoing iterative revisions to protocols, workflows, and sustainability of CHESS.

Through the process of working with staff champions to identify patient communication opportunities, multiple points for providing CHESS were identified in both institutions. These points included the following: (1) at or shortly after diagnosis by care coordinators or nurse navigator by phone, (2) during initial clinic visits with surgeons and care coordinators/nurse navigator, (3) during radiation or chemotherapy treatments, and (4) upon consultation with additional staff such as the oncology social workers or the American Cancer Society (ACS) Navigator, located on-site at KPCO. Stakeholders from each of these groups provided input into the tools required to make CHESS introduction easy and accessible for physicians, staff, and patients. Tools and processes for introducing CHESS were also customized to each organization. For example, some stakeholders suggested that women would want information about their treatment and what comes after while having infusion treatment and therefore resources for the infusion rooms would be helpful. To meet this need, iPads were provided to both institutions preloaded with shortcuts to CHESS. At one institution, multiple stakeholders were very committed to providing resources for all cancer patients, not just breast cancer patients, and continually expressed concerns that CHESS was a resource only for breast cancer. Therefore, the iPads provided to the clinics were also preloaded with shortcuts to other trusted, accurate, general cancer sites (American Cancer Society, National Cancer Institute, Livestrong). Infusion clinic staff developed protocols for checking out iPads and were reminded that the iPads were available for use by all cancer patients.

One site requested signage for all exam rooms, brochures to be available in radiation oncology and oncology waiting rooms, and fliers and business cards for display and inclusion in patient information packets. The site with concerns over CHESS being a resource only for breast cancer patients did not want signs or fliers in waiting rooms and felt that business card-sized materials would be lost by patients and therefore only requested fliers that could be included in patient information packets. This site did, however, have another mode for sharing information about CHESS. Because of the electronic health record system (EHR), this site requested smart text and phrases (template language) that could be used to add an electronic link to CHESS through patient emails, letters, after visit summaries, and chart notes (which are accessible to patients through the patient portal). These template text messages also included a live link to the CHESS registration page, allowing patients accessing the information through the patient portal of the EHR to directly access CHESS by clicking on the live link.

As CHESS implementation continued, it became clear that some aspects of CHESS were a better fit with the two organizations than others, and that some stakeholders were less involved or had less opportunity to introduce CHESS than others. For example, at both sites, most patients were offered CHESS only by the general surgery care coordinators or the nurse navigator who speaks to patients shortly after diagnosis. These staff members provide CHESS access information via telephone or in an email around that initial 
phone call, they also include a CHESS flier in the information packet they provide to all breast cancer patients at the first visit. At both sites, some physicians introduce CHESS using the EHR tools (at KPCO) or printed resources (EHC). For patients who come to the EHC system later in the treatment process, the social worker, psychologist, and some physicians provide CHESS information as a flier, business card, or simply point out the poster in the exam room. At both institutions, iPads were available in the infusion centers, though most iPad use turned out to be for entertainment rather than CHESS or other breast cancer websites.

Tracking formal and informal communications throughout the implementation period has resulted in the research team learning about organizational context changes that could affect the implementation of CHESS, the fit of CHESS with values and needs, the continued alignment of CHESS with current care processes, and the future sustainability of CHESS as part of standard care. For example, from October 2011 through August 2013, both organizations experienced at least one major departmental restructuring, staffing turnover, and/or leadership reorganization.

Minor changes at each organization were made to the information packets provided to patients and how CHESS was introduced across the continuum. At $\mathrm{KPCO}$, soliciting feedback from infusion staff during the formal structured interviews resulted in CHESS being more openly offered during treatment through a flier that could be kept behind the desk and handed to a patient. Furthermore, continued informal communication with oncology managers resulted in opportunities for future dissemination of CHESS at both sites. As an embedded clinician, the CHESS site lead at EHC is also a clinical leader, which has provided an opportunity for future dissemination of CHESS as standard care at additional EHC hospitals in the Denver area. Informal communications with the oncology manager at $\mathrm{KPCO}$ resulted in multiple conversations with a national group working to improve breast cancer care across all Kaiser Permanente regions.

\section{DISCUSSION}

Implementing evidence-based innovations into standard care in large health care organizations is a complex undertaking dependent upon many different factors. We report on an attempt to implement an ICCS, breast cancer CHESS, into standard care for breast cancer patients in a systematic, theoretically guided process led by experts in ICCS development and evaluation, as well as in dissemination and implementation research. What we have learned from this longitudinal observational study of breast cancer CHESS implementation at $\mathrm{KPCO}$ and $\mathrm{EHC}$ has implications for implementing other eHealth innovations at healthcare organizations (Table 1). Table 1 provides a summary of our conclusions and solutions by theme with lessons learned as guidance for future implementation efforts for other organizations.
Overall, it is clear that innovations must be compatible with organizational priorities and needs, must show an advantage over current practice to most if not all stakeholders, must not be overly complex for the system, must be flexible to respond to healthcare changes, and must show results observable by the stakeholders $[16,17]$. CHESS had shown relative advantage over standard care and was simple enough to implement through recommendation to patients. For implementation at $\mathrm{KPCO}$ and $\mathrm{EHC}$, stakeholders were involved to ensure compatibility with organizational priorities and needs. In short, implementation of CHESS followed the principles of designing for dissemination and implementation [18]. However, organizational priorities are not static; stakeholders change and stakeholder opinions and experiences can change during the trial period. This is not to say that implementation of new innovations and dissemination throughout large health care organizations is impossible, but rather, that successful implementation requires continuous compromises and adjustments, especially when trying to align research grant priorities and timelines with clinical and organizational timelines and priorities. These concessions also require a shift from the traditional paradigm of linear translation of a predesigned product (the intervention developed through RCTs) through centralized change agents, to a paradigm where innovations are malleable with input from researchers and the end adopters (clinicians and staff) who can make insightful real-time improvements [19, 20]. Additionally, if knowledge sharing can be facilitated among implementing staff then lessons about best practices, reasons for failure, and solution-providing champions can further affect the process of organizational learning through additional improvement [21, 22].

We observed that as clinical stakeholders were reengaged to participate in the implementation of CHESS, the website as previously developed and tested required adaptation, even prior to its implementation. Innovations often require reinvention or adaptation to fit the local conditions and suit the needs of the setting [17, 23], particularly as technology evolves rapidly. Recent commentary also suggests that rather than viewing the contextual factors as altering the effectiveness of the innovation as designed, these factors should be regarded as leading to beneficial adaptations that improve fit of the innovation with the setting and improve sustainability of the innovation within the organization $[24,25]$. In this case, the redesign of CHESS resulted in a better fit with busy physician schedules by removing the need for continual physician review to ensure accuracy of material, which also improves the sustainability of CHESS within the system because minimal organizational resources and clinician involvement are required for maintenance.

Communication between the study team and implementing staff is clearly important to implementation. First of all, if there is to be any lag in timing from initial stakeholder engagement to actual start of implementation processes, continued engagement with multiple stakeholders is necessary to both remind those 
stakeholders about the program and to ensure continued fit with their priorities, needs, and values. We found that physicians and staff had an "out of sight, out of mind" experience during the months between initial stakeholder meetings for engagement and grant writing and the time that implementation began, resulting in repetition of previous conversations about why CHESS was chosen and how it fit with their values, priorities, and needs. Although this was a surprise to the research team, in hindsight this should be expected as physicians are busy with everyday patient care and may have little capacity for issues that are not immediately relevant. Due to organizational restructuring and staff changes, maintaining contact with only one key stagehold was not sufficient for us to adjust prospectively to changes and their impact on implementation.

Second, framing innovations as a trial or "try before you buy" program may have value to some stakeholders who are concerned with advantage over current care, compatibility, complexity, or results of the innovation's effectiveness in their environment. We found that this framing helped some stakeholders perceive limits to their commitment to CHESS adoption. This is consistent with the perceived attribute of trialability of innovation [17] and addresses the differences in individual adopters' degree of innovativeness $[17,18]$ or readiness to change [26]. This framing, along with the assurance of organizational maintenance, allowed early adopters and individuals in the ready-to-change stage to feel confident that the CHESS resource would remain available once adopted and allowed the later adopters or contemplators to feel comfortable that CHESS could be discontinued if they did not feel it was useful or if CHESS ceased to fit with the environment.

Finally, constant avenues for formal and informal communication were found to be critical to maintain alignment with organizational values and needs. This communication also served as a venue for showing effects of the CHESS resource over time and allowed for changes, solutions, and explanations of observed effects in real-time. The informal communication with clinic leaders led to opportunities to explore further CHESS expansion within both organizations.

While each healthcare system is unique, it is well known that physician support is critical for implementing an innovation in the healthcare setting. Yet many aspects of clinician culture are known barriers to gaining this support, such as a professional norm that resists improvement efforts presented by external stakeholders [27], as well as physician time and competing demands [27, 28]. One solution to physician resistance may be finding additional champions at the level that is most appropriate for the institutional processes of who would be actually responsible daily for the intervention, whether it is physician or staff. These champions may be helpful in developing processes and promoting the innovation as issues of compatibility, complexity, and alignment are assessed and addressed. In this case example, we observed that initially reluctant physicians were eventually supportive and accepting of CHESS as standard care, but they lacked the time to be champions of the intervention and to develop processes to consistently offer CHESS to patients. However, champions became apparent in the staff that expressed the most need for CHESS and who expressed a willingness to incorporate it into processes to ensure every newly diagnosed breast cancer patient was offered access to CHESS. These same champions also made it clear that CHESS, or any resource like it, at best augmented rather than displaced the important work that they themselves did in helping breast cancer patients.

Additional factors affecting implementation may include the size of the organization and the embeddedness of research staff. EHC is about one third the size of the KPCO oncology practice, increasing the patient load placed on providers and creating different care environments at the two institutions. Size also places more levels of management between physicians, staff, and organizational leaders. Likewise, at EHC, the site leader and champion is a clinician embedded within the care delivery system, whereas at KPCO the researchers are in a department within the system but external to the clinical care environment. This difference could also affect the implementation and dissemination of CHESS and other innovations, as the embedded clinician has more face-time with other clinicians and can serve as a constant peer champion of the innovation to other clinicians, staff, and leaders. When researchers are not embedded in the clinical practice, communication opportunities must be crafted to maintain visibility of the innovation and support its relevance to the daily care of patients. This observation is also consistent with the argument that researcher-led implementation efforts address gaps important to the researchers, but not necessarily important to the clinical staff [27]. Having the implementation lead as a clinician embedded within the system may also remove this perceived disconnect when the implementation is led by external individuals.

Some of the observed initial resistance to CHESS may also have been part of an innate cultural difference between an integrated system and a fee for service system. An integrated system may be more inclined to create its own internal processes and solutions to meet the needs of both patients and the organization, which is both the care provider and the insurer. In contrast, the culture in fee for service organizations may be more apt to take advantage of any existing/external resources that can improve the organization's value in the eyes of the patient. Additionally, assessment of "acceptable evidence" to implement an innovation may differ because of different economic effects resulting from the system not being both the insurer and the care provider.

In conclusion, it is clear from this observational case study that implementation of an ICCS such as breast cancer CHESS as part of standard care in nonacademic clinical settings is not trivial, nor can it be taken lightly. This implementation test had a number of factors in place to facilitate successful implementation: 
a fully developed and well-tested eHealth tool which fit with evidence-based principles of the organizations, a team with extensive theoretical and practical expertise both with that tool and in dissemination and implementation of health innovations, progressive healthcare organizations willing to attempt implementation at the leadership level, a systematic process of engaging stakeholders, and general and organizationspecific knowledge of how large healthcare organizations work and change.

Despite these facilitators and the experience of the implementation study team, many factors that cannot be controlled still affected implementation. Organizational priorities and changes affect clinical staff involved in the implementation, and if the leaders are not directly involved in implementation, the innovation will not be considered a factor in any changes being made. Other factors contributing to differences in implementation may also include size of the patient population, clinically embedded vs. non-embedded researchers, and the type of system (integrated vs. fee for service). Implementation, therefore, requires the anticipation of as many factors as possible and the ability to adjust as necessary to organizational and individual factors that are unforeseen and usually not in the control of the implementers. Creating processes for constant formal and informal communication along with objective tracking of events affecting implementation can help with the process of implementation and dissemination of interventions throughout any organization. Finally, observational case studies, such as this one, provide much needed insights into the reasons why certain strategies may or may not work in different settings, and also to confirm that compatibility of the innovation to staff, organizations, and patients' preferences, beliefs, and practices are especially important factors affecting implementation.

Acknowledgments: This was an investigator-initiated study funded through National Institute of Health (NIH) grant no. 1R01CA149005-01A1. The NIH played no role in the design, conduct, or analysis of the study and interpretation of findings. All authors had full access to data and take responsibility for the integrity of the data and accuracy of the analysis.

Conflict of interest: The authors Alanna Kulchak Rahm, Robert P. Hawkins, James W. Dearing, Suzanne Pingree, Jana Bolduan Lomax, Helen McDowell, Erica Ferro Morse, and BreAnne Barela declare that they have no conflict of interest; no financial relationships with any organizations that might have an interest in the submitted work; and no other relationships or activities that could appear to have influenced the submitted work. The authors also state that they have full control of all primary data, and that they agree to allow the journal to review their data if requested.

Adherence to ethical principles: This study was reviewed and approved by the Institutional Review Boards (IRB) at the University of Wisconsin Madison, Exempla Saint Joseph Health System, and Kaiser Permanente Colorado.

1. Fox S. The Engaged E-patient Population: Report of the Pew Internet and American Life Project. http://www.pewinternet.org/Reports/ 2008/The-Engaged-Epatient-Population.aspx. 2008.
2. Gustafson DH, Hawkins R, McTavish F, et al. Internet-based interactive support for cancer patients: are integrated systems better? J Commun. 2008; 58(2): 238-257.

3. Gustafson DH, Hawkins RP, Boberg EW, et al. CHESS: 10 years of research and development in consumer health informatics for broad populations, including the underserved. Int / Med Inform. 2002; 65(3): 169-177.

4. Gustafson DH, Hawkins R, Pingree S, et al. Effect of computer support on younger women with breast cancer. J Gen Intern Med. 2001; 16(7): 435-445.

5. Gustafson DH, Hawkins R, Boberg E, et al. Impact of a patientcentered, computer-based health information/support system. Am J Prev Med. 1999; 16(1): 1-9.

6. Gustafson DH, McTavish FM, Stengle W, et al. Reducing the digital divide for low-income women with breast cancer: a feasibility study of a population-based intervention. J Health Commun. 2005; 10(Suppl 1): 173-193.

7. Gustafson DH, McTavish F, Hawkins R, et al. Computer support for elderly women with breast cancer. JAMA. 1998; 280(15): 1305.

8. Murray E, Burns I, See TS, Lai R, Nazareth I. Interactive Health Communication Applications for people with chronic disease. Cochrane Database Syst Rev. 2005; (4):CD004274.

9. Gysels M, Higginson IJ. Interactive technologies and videotapes for patient education in cancer care: systematic review and metaanalysis of randomised trials. Support Care Cancer. 2007; 15(1): 7-20.

10. Strecher V. Internet methods for delivering behavioral and healthrelated interventions (eHealth). Annu Rev Clin Psychol. 2007; 3: $53-$ 76.

11. Gustafson DH, DuBenske LL, Namkoong K, et al. An eHealth system supporting palliative care for patients with non-small cell lung cancer: a randomized trial. Cancer. 2013; 119(9): 1744-1751.

12. Hawkins RP, Pingree S, Baker T, et al. Integrating eHealth with human services for breast cancer patients. Transl Behav Med. 2011; 1(1): 146-154.

13. Gustafson D, Brennan P, Hawkins R. Investing in E-Health: What it Takes to Sustain Consumer Health Informatics. New York: Springer; 2007.

14. Van de Ven AH, Polley DE, Garud R, Venkataraman S. The Innovation Journey. Oxford University Press, Inc; 1999.

15. Zmud RW. An examination of "push-pull theory" applied to process innovation in knowledge work. Manag Sci. 1984; 30: 727-738.

16. Dearing JW. Evolution of diffusion and dissemination theory. J Public Health Manag Pract. 2008;14(2).

17. Rogers E. Diffusion of Innovations. 5th ed. New York: The Free Press; 2003.

18. Dearing JW, Kreuter MW. Designing for diffusion: how can we increase uptake of cancer communication innovations? Patient Educ Couns. 2010; 81(Suppl): S100-S110.

19. Dearing JW, Kee KF. Historical roots of dissemination and implementation Science. In: Brownson RC, Colditz GA, Proctor EK, eds. Dissemination and Implementation Research in Health. New York: Oxford University Press, Inc; 2012: 55-71.

20. von Hippel E. Democratizing Innovation. Cambridge, MA: The MIT Press; 2005.

21. Dearing JW, Greene SM, Stewart WF, et al. If we only knew what we know: principles for knowledge sharing across people, practices, and platforms. Transl Behav Med. 2011; 1(1): 15-25.

22. Schilling L, Dearing JW, Staley P, et al. Kaiser Permanente's performance improvement system, Part 4: creating a learning organization. Jt Comm J Qual Patient Saf. 2011; 37(12): 532-543.

23. World Health Organization. Practical Guidance for Scaling Up Health Service Innovations. Geneva, Switzerland: World Health Organization Press; 2009.

24. Chambers DA, Glasgow RE, Stange KC. The dynamic sustainability framework: addressing the paradox of sustainment amid ongoing change. Implement Sci. 2013; 8(1): 117.

25. Dearing JW, Meyer G. The Active Adopter in the Diffusions of Innovations. The Diffusion of Innovations: A Communication Science Perspective. New York: Peter Lang; 2011: 207-230.

26. Prochaska JO, DiClemente CC. The transtheoretical approach. In: Norcross JC, Golfried MR, eds. Handbook of Psychotherapy Integration. 2nd ed. New York: Oxford University Press; 2005.

27. Mittman BS. Implementation Science in health care. In: Brownson RC, Colditz GA, Proctor EK, eds. Dissemination and Implementation Research in Health. New York: Oxford University Press, Inc; 2012: 400-418.

28. Price DW, Miller EK, Rahm AK, et al. Assessment of barriers to changing practice as CME outcomes. I Contin Educ Health Prof. 2010; 30(4): 237-245. 